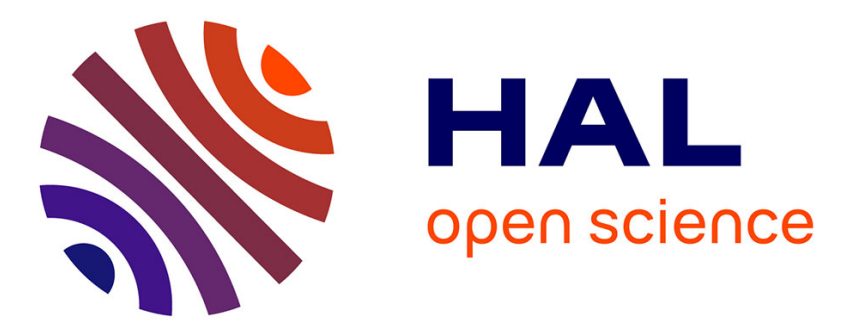

\title{
Transient Internal Damping in Metal Matrix Composites: Experiment and Theory
}

\author{
A. Vincent, G. Lormand, S. Durieux, Christian Girard, E. Maire, R. Fougères
}

\section{To cite this version:}

A. Vincent, G. Lormand, S. Durieux, Christian Girard, E. Maire, et al.. Transient Internal Damping in Metal Matrix Composites: Experiment and Theory. Journal de Physique IV Proceedings, 1996, 06 (C8), pp.C8-719-C8-730. 10.1051/jp4:19968157 . jpa-00254591

\section{HAL Id: jpa-00254591 https://hal.science/jpa-00254591}

Submitted on 1 Jan 1996

HAL is a multi-disciplinary open access archive for the deposit and dissemination of scientific research documents, whether they are published or not. The documents may come from teaching and research institutions in France or abroad, or from public or private research centers.
L'archive ouverte pluridisciplinaire HAL, est destinée au dépôt et à la diffusion de documents scientifiques de niveau recherche, publiés ou non, émanant des établissements d'enseignement et de recherche français ou étrangers, des laboratoires publics ou privés. 
JOURNAL DE PHYSIQUE IV

Colloque C8, supplément au Journal de Physique III, Volume 6, décembre 1996

\title{
Transient Internal Damping in Metal Matrix Composites: Experiment and Theory
}

\author{
A. Vincent, G. Lormand, S. Durieux, C. Girard, E. Maire and R. Fougères
}

Groupe d'Etude de Métallurgie Physique et de Physique des Matériaux, UMR 5510 du CNRS, INSA, Bât. 303, 69621 Villeurbanne cedex, France

\begin{abstract}
In this presentation, first, the Internal Damping (I.D.) phenomena and related theories that have been previously reported in the literature for Metal Matrix Composites (M.M.C.s) are reviewed briefly. Then, the paper is focused on the transient ID phenomena. Especially, it is shown that a broad low temperature peak appears in the low frequency internal damping (ID) spectra of aluminium based microheterogeneous materials. The role of the various experimental and material parameters is presented. Concerning material parameters, in addition to the influences of the matrix microyield stress and volume fraction of reinforcing particles, the role of particle - matrix interfacial region is carefully investigated.

From the overall experimental observations it is proved that the transient ID phenomena are linked with emission and movement of dislocations around particles, which results from the micromechanical interaction between particle and matrix. Moreover, assuming a perfect particle-matrix interface, a dislocation model has been developed in order to account quantitatively for the observed phenomena. The predictions of the model agree fairly well with the experimental behaviour of MMCs for which the assumption of a perfect interface is expected to be sound. Finally, the behaviour of MMCs with modified interfacial regions is discussed, using the above mentioned model, in order to identify the mechanism liable for the differences between the corresponding ID spectra.
\end{abstract}

\section{INTRODUCTION}

Metal Matrix Composites (M.M.C.s), which consist of additioning ceramic reinforcements to a metallic alloy, have received a great deal of interest for more than ten years, due to the practical and academic importances of such a concept. However, the mechanical behaviour of M.M.C.s exhibits many special, expected or unexpected, characteristics concerning elastic modulus, damping capacity, microyield and yield stresses, plasticity, mechanical and thermomechanical fatigue strengths, which are not yet completely understood. As explained in some basic papers $[1,2]$ many of these specific properties arise from the concept itself : introducing particles or fibres in a metal matrix with quite different mechanical and thermal properties results in inhomogeneities in the strain and temperature fields as the M.M.C. is subjected to a mechanical or thermal loading. Another key point for the M.M.C. properties is the reinforcement-matrix interface, which should ensure the load transfer from the matrix to the reinforcement. Unfortunately, up to now, few experimental data about the behaviour of the reinforcementmatrix interface and the neighbouring matrix have been made available. Only T.E.M. observations have provided information about microstructural changes at the interface or around particles $[3,4,5]$.

In the past, various aspects of M.M.C. behaviour have been investigated by means of Internal Damping (I.D.) studies, either in view of developing materials with enhanced damping properties or, more often, in order to characterize the interaction mechanisms between reinforcing particles and matrix, which, as mentioned above, play a fundamental role in the M.M.C.s mechanical properties. In this presentation, first, the ID phenomena and related theories that have been previously reported in the literature are reviewed briefly. Then, the paper is focused on the transient ID phenomena. The role of the various experimental and material parameters is presented. Concerning material parameters, in addition to the influences of the matrix microyield stress and volume fraction of reinforcing particles, the role of the particle-matrix interfacial region has been carefully investigated. In the next section, a model for computing I.D. vs temperature is briefly presented. Finally the predictions of the model are discussed and compared with some selected experimental results. 


\section{BACKGROUND ON M.M.C.S AND RELATED INTERNAL DAMPING}

As mentioned previously, most specific properties of M.M.C.s result from the quite different mechanical and thermal characteristics of ceramic reinforcement and metal matrix. These different properties generate marked inhomogeneities in the stress and temperature fields when the M.M.C. is subjected to a mechanical or thermal loading. This section aims at summarizing how the stress field is built up around reinforcements in M.M.C.s and then reviewing briefly how the resulting inhomogeneties in the stress and temperature fields may be responsible for specific I.D. phenomena.

\subsection{The stress field inhomogeneity}

The difference between the properties of ceramic reinforcement and metallic matrix is responsible for a strain mismatch when the M.M.C. is mechanically or thermally loaded. As a result, an inhomogeneous stress field is built up in and around the reinforcement to accommodate this strain mismatch. For simple situations, i. e. spherical or ellipsoidal particles with particles and matrix perfectly bounded, the stress field around a single microheterogeneity in an infinite matrix can be calculated easily as long as the system behaves elastically. Such calculations can be achieved by various methods such as the finite element method or the Eshelby method. Moreover, for few configurations with a high degree of symmetry, completely analytical solutions can be found.

Typical stress fields obtained by means of the Eshelby method [6], which is advantageous because it does not require a careful meshing around the reinforcing particle, called inclusion in [6], are given in Fig. 1. The situation for a pure thermal stress field around a spherical particle is shown in Fig. 1-a. This situation is met when a system is cooled down from a temperature $T_{0}$ for which it was initially free of stress. Upon cooling the system a strain mismatch is then developed, due to the difference between Coefficients of Thermal Expansion (C.T.E.s) of inclusion and matrix. This strain mismatch $\varepsilon_{\mathrm{m}}$ at a lower temperature $\mathrm{T}$ is $: \varepsilon_{\mathrm{m}}=\Delta \alpha \Delta \mathrm{T}$, where $\Delta \alpha$ is the difference of C.T.E. between inclusion and matrix and $\Delta \mathrm{T}=\mathrm{T}_{\mathrm{o}}-\mathrm{T}$.

a)

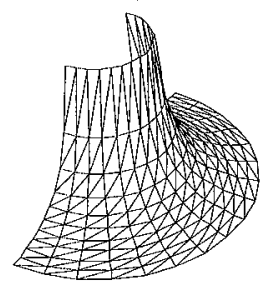

b)

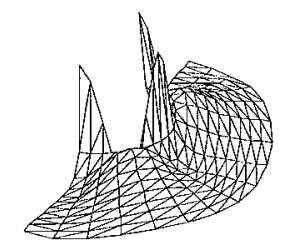

c)

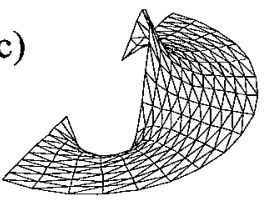

Figure 1 : Local stress distribution around a SiC spherical particle in an Al matrix, in a plane cutting the particle through its centre; Young's moduli used for the calculations are $70 \mathrm{GPa}$ and $400 \mathrm{GPa}$ for Al and SiC, respectively ; thermal expansion coefficients are $3.510^{-6}$ and $23.510^{-6}$, for $\mathrm{Al}$ and $\mathrm{SiC}$, respectively ; a) shear stress resulting from a pure thermal loading ; b) and c) local stress resulting from an applied shear stress , b) shear stress and c) hydrostatic component of the stress tensor.

It is worth noting the shear stress in the matrix varies as $\varepsilon_{\mathrm{m}}$. Furthermore, the stress inhomogeneity that results from a mechanical loading is illustrated in Fig. 1-b-c, for a SiC spherical particle embedded in an aluminium matrix. The case of an applied shear stress has been chosen since materials tested in a torsion pendulum are submitted to such a type of loading. Thus, Fig. 1-b shows that the shear stress is maximum at four positions at $90^{\circ}$ to each other along the equator circle of the sphere. It should be noted that the stress concentration factor, i.e. maximum shear stress around particle over applied shear stress, amounts to 1.86 for the studied system. Besides, Fig. 1-c shows that the hydrostatic component of the stress is alternately maximal and minimal at positions situated at mid-distance between the pole and the maxima of the shear stress shown in Fig. 1-b.

In brief, these results illustrate the fact that marked inhomogeneities in the stress field result from the differences in the M.M.C.s properties. Then, specific I.D. may result from such an inhomogeneous stress field as soon as some irreversible process is activated in the material to relax the stress concentration. This may occur by plastic flow associated with dislocation glide in the matrix, atomic diffusion, debonding and glide along the reinforcement-matrix interface or cracking in the matrix. In fact, it should be kept in mind that in some cases the process is not so straightforward, but it may involve two steps. Namely, the 
relaxation process occurs prior to the I.D. measurement, during specimen elaboration or mechanical loading, so that a particular microstructure is built up or damage is created around reinforcements. Such a microstructure or a damage is then investigated by means of I.D. measurements. The following part of this section aims at reviewing the various mechanisms that have been reported in the literature.

\subsection{Dislocations induced by the C.T.E. mismatch - Related I.D.}

In this section, first the process of creation of dislocations induced by the C.T.E. mismatch is briefly described and then I.D. studies related with such dislocations are reviewed.

Consider the situation in which the M.M.C. is initially free of stress at the temperature of thermal treatment. On cooling the material the thermal stress increases rapidly with decreasing the temperature (see section 2.1). For high strength particles well bonded to the matrix, it is well established that, when a critical stress is reached, stress relaxation occurs by dislocation punching out from the particle/matrix interface [7]. The resulting high density of dislocations around reinforcements has actually been observed by various authors [3]. Among the various models that have been proposed for describing this plastic zone $[7,8]$, let us describe the bases of the method proposed by Hamann and Fougères [9] that enables one to determine the positions of dislocation loops around a single spherical particle embedded in an infinite matrix throughout the cooling process. It should be noted that the model is soundly applicable for a finite concentration of particles as long as the plastic zones around neighbouring particles do not overlap. This model will be used as a basis for modelling I.D., as presented in section 3 of this paper.

As mentioned above, when the matrix yield stress is reached, thermal stresses can be, at least partly, relaxed by punching out of dislocation loops from particle-matrix interface. In the Hamann's approach, the scheme proposed by Hull and Bacon [10] has been adopted, namely it is assumed that loops are punched out symmetrically along all $12<110>$ directions on glide cylinders intersecting the spherical particle along circles where the resolved shear stress is maximum. Then, in the Hamann's approach the dislocations are treated by groups of 12 prismatic loops. Moreover, the dislocation glide is assumed to be controlled by a friction stress that represents interactions of dislocations with obstacles opposing their motion. This friction stress is assumed to be independent of the dislocation velocity. Moreover, the local strain hardening of the matrix due to the increase in dislocation density around the particle has been accounted for by considering, in the equilibrium equations, a friction stress given by the classical equation: $\tau_{\mathrm{f}}(\mathrm{x})=\tau_{\mathrm{fo}}+\beta \mathrm{Gb} \sqrt{\Lambda(\mathrm{x})}$, where $\Lambda(\mathrm{x})$ is the density of dislocation at a distance $\mathrm{x}$ from the particle, $\tau_{\mathrm{fo}}$ is the intrinsic friction stress of the matrix whose temperature dependence is also taken into account, $G$ the shear modulus of the matrix, $b$ the Burgers vector of dislocations in the matrix and $\beta$ a strain hardening coefficient whose value is between 0.3 and 0.6 for metals.

Hence, the process may be described according to the following steps. First, the dislocation punching out from the particle-matrix interface is expected to occur when the local effective driving shear stress at the interface, $\tau_{\mathrm{d}}(0)$, exceeds the local friction stress $\tau_{\mathrm{f}}(0)$, that is :

$$
\tau_{\mathrm{d}}(0)>\tau_{\mathrm{f}}(0)
$$

Second, once a dislocation set has been emitted, it moves away, at a distance of the particle hereafter noted $x^{0}$, until the driving stress acting on the loops, $\tau_{d}\left(x^{0}\right)$, becomes equal to the local friction stress $\tau_{\mathrm{f}}\left(\mathrm{x}^{0}\right)$. The punching distance for the first group of emitted loops is thus deduced from the equation :

$$
\tau_{\mathrm{d}}\left(\mathrm{x}^{0}\right)=\tau_{\mathrm{f}}\left(\mathrm{x}^{0}\right)
$$

Finally, upon further cooling the system, successive groups of dislocations are emitted. The conditions for punching out and positions reached by dislocations are similar to equations (1) and (2) but now the interaction between various groups of dislocations must be taken into account. The detail of the calculation are given in reference [9]. An example of the dislocation distribution predicted by such an approach is shown in Fig. 2 , where the positions of successively emitted dislocation groups have been plotted vs temperature for a SiC spherical particle of radius $\mathrm{a}=5 \mu \mathrm{m}$, in a 7075 Al alloy matrix cooled from $430 \mathrm{~K}$ to $100 \mathrm{~K}$. In this figure, two domains can be recognized. First, in the temperature range $430<\mathrm{T}<320 \mathrm{~K}$ the matrix remains elastic. Second, on further decreasing temperature, punching out of dislocation groups occurs with a quasi constant temperature decrement between successive events. At the lowest temperature, $100 \mathrm{~K}$, the total number of emitted groups is 25 and the maximal punching out distance is $2.2 \mu \mathrm{m}$. 


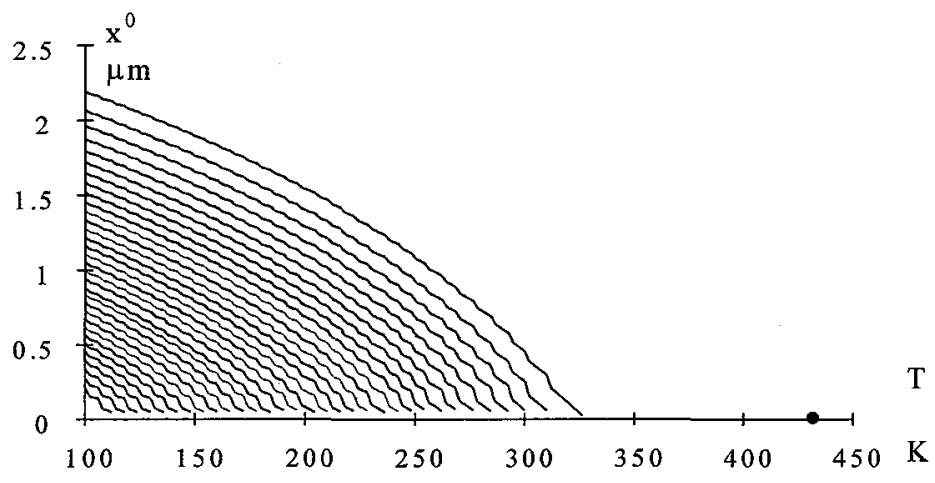

Figure 2 : Distance $\mathrm{x}^{0}$ from the particle, of successively emitted dislocations groups, vs $\mathrm{T}$ on cooling a $7075 \mathrm{Al}$ alloy/SiC composite from $430 \mathrm{~K}$ ( $\bullet$ stress free temperature) to $100 \mathrm{~K}$.

Various works dealing with I.D. linked with such dislocations induced by the C.T.E. mismatch have been reported in the literature. A first series of studies is concerned with dislocations created around reinforcing particles during the elaboration process, i.e. prior to the I. D. measurement. Then, during the I.D. measurement, a classical dislocation damping mechanism is activated by the measurement stress that is locally amplified. The type of mechanism depends on the material and the temperature range that are investigated. For instance, in Al based composites, around room temperature, dislocation breakaway mechanisms may operate $[11,12]$, thus leading to a typical strain amplitude dependence of I.D., whereas at high temperature relaxation mechanisms by cross-slip are more likely to be active [13]. Besides, the ultrasonic low-frequency stress-coupling technique has also been used successfully [14]. In such experiments, the dislocation breakaway process is induced by a low amplitude bias stress, and the resulting attenuation change is used as an indicator of the mobility and density of dislocations. Then, the emission and the movements of dislocations are evidenced through the marked evolutions of attenuation changes, during a thermal cycle.

A second type of phenomenon is concerned with dislocation motions induced by the C.T.E. mismatch during the I.D. measurement itself. That occurs when the measurements are carried out during the temperature variation, either on cooling or heating the specimen. The resulting coupling between the local microplastic strain of thermal origin and the oscillating shear stress produced by the pendulum oscillations is responsible for a specific I. D.. It is worth noting the process is very similar to that involved around first order phase transitions in solids $[15,16]$. To our knowledge, this phenomenon was first observed in Al-Si alloys that are composite like [17], and more recently in M.M.C.s.[18, 19]. This process will be presented in detail in sections 3,4 and 5 of this paper.

\subsection{I.D. caused by atomic diffusion around reinforcing particles}

As mentioned above, relaxation of the stress concentration around a reinforcing particle may occur by means of atomic diffusion that tends to compensate the matrix-particle strain misfit. That is, atoms are expected to move from the compressional to the dilatational regions shown in Fig. 1.c, and conversely for vacancies. When, in an I.D. apparatus, the relaxation time of the process is of order of the period of oscillation, it is expected to produce a large energy loss, that is an I.D. peak vs temperature. Such a welldeveloped I.D. peak was observed by Okabe et al [20] in Al-Si alloys, in the temperature range 400-450 $\mathrm{K}$, for a period of oscillation of $1 \mathrm{~Hz}$. This peak was unambiguously attributed to the Si particles, whose diameter was in the range $50-60 \mathrm{~nm}$. It should be noted that, according to the theory proposed by Okabe et al, the relaxation time would vary as the power 3 of the particle diameter, that is the peak temperature would be situated at very high temperature for M.M.C.s with large ceramic particles.

\subsection{I.D. related with stress relaxation by matrix-reinforcement debonding}

Matrix-reinforcement debonding is a possible process to relax the stress concentration around particles, which is likely to occur for high strength matrices and poor quality interfaces. Parrini and Schaller [21] have evidenced such a process by means of I.D. measurements vs strain amplitude, in aluminium based composites reinforced with alumina short fibres. Thus, I.D. vs strain amplitude exhibits a marked peak, whose characteristics are those of a solid friction peak. The overall observed properties enabled the authors to ascribe this peak to the beginning of debonding along the reinforcement-matrix interface. 


\subsection{I.D. related to temperature field inhomogeneity}

When a material region is subjected to a rapid change of stress, there is a corresponding change in the temperature in that region. This phenomenon is known as the thermoelastic effect. Hence, when a M.M.C. is loaded, an inhomogeneous temperature field may result from the inhomogeneous distribution of the stress as well as from the differences between matrix and reinforcement properties. Consequently, irreversible heat conduction occurs from high temperature regions to low temperature ones thus producing entropy, which results in I.D. This process was first studied by Zener in the case of a homogeneous beam submitted to flexural vibrations. More recently, I.D. induced by the specific inhomogeneities expected in composite materials has been treated theoretically by Kinra in some simple configurations [22, 23$]$.

\section{EXPERIMENTAL RESULTS}

\subsection{Material and experimental method}

A part of the study was carried out on a 7075 aluminium alloy reinforced by SiC particles. The average size of the particles was of order of $10 \mu \mathrm{m}$ and their volume fraction was $15 \%$. The composite was produced by the Osprey route (ALCAN supplier). Then it was submitted to a T7651 treatment : solution treated at $470^{\circ} \mathrm{C}$ for $4 \mathrm{~h}$, quenched, and then aged for $6 \mathrm{~h}$ and $24 \mathrm{~h}$ at $120^{\circ} \mathrm{C}$ and $160^{\circ} \mathrm{C}$, respectively.

An other part of the study was carried out on MMCs elaborated by the powder metallurgy process, from pure $\mathrm{Al}$ powder and $\mathrm{SiC}$ particles with an average size of $10 \mu \mathrm{m}$. All the MMCs have been HIP treated at $525^{\circ} \mathrm{C}$ under $200 \mathrm{MPa}$ for $4 \mathrm{~h}$. On the one hand, some materials were produced with various volumetric fractions of $\mathrm{SiC}$ particles. On the other hand, in order to investigate the role of the $\mathrm{Al} / \mathrm{SiC}$ interfacial region on $\mathrm{ID}$, the interfacial regions of these $\mathrm{Al} / \mathrm{SiC}$ composites have been modified by changing the elaboration conditions as follows : i) for the standard material the $\mathrm{SiC}$ particles were used as received ; ii) for another M.M.C., prior to the elaboration of the composite, the SiC particles were oxidized for $1 \mathrm{~h}$ at $1200^{\circ} \mathrm{C}$ in a dry atmosphere ( $\mathrm{MMC}$ hereafter called OX1) ; iii) for a third one the oxydization was carried out at $1000^{\circ} \mathrm{C}$ in wet atmosphere (OX2).

All the specimens for I.D. measurements were electrically discharge machined. They had a gauge length of $48 \mathrm{~mm}$ and a cross section of $5 \times 0,3 \mathrm{~mm}^{2}$.

The I.D. measurements were performed on a computer controlled torsion pendulum of inverted type. The oscillation frequency $\mathrm{f}$ of this pendulum is in the range $0.3<\mathrm{f}<2 \mathrm{~Hz}$ with the above described specimens. The system is initially forced to oscillate at an imposed amplitude, and then left to oscillate freely. The I.D. of the specimen is characterized by the logarithmic decrement of the freely decaying oscillations. The measurements were generally carried out in the temperature domain $430-100 \mathrm{~K}$, with various cooling rates in the range $50-200 \mathrm{~K} / \mathrm{h}$.

\subsection{I.D. during cooling - influence of cooling rate, frequency and strain amplitude}

The I.D. spectra for the 7075/SiC composite are presented in Fig. 3. A typical I.D. spectrum (e.g. curve "a"), recorded on continuously cooling the specimen, exhibits a rapid decrease of the high temperature background and then a broad Low Temperature Maximum (L.T.M.) situated around $140 \mathrm{~K}$. A noticeable feature of the L.T.M. is that it is strongly reduced as the cooling rate is decreased from 200 to $50 \mathrm{Kh}^{-1}$.

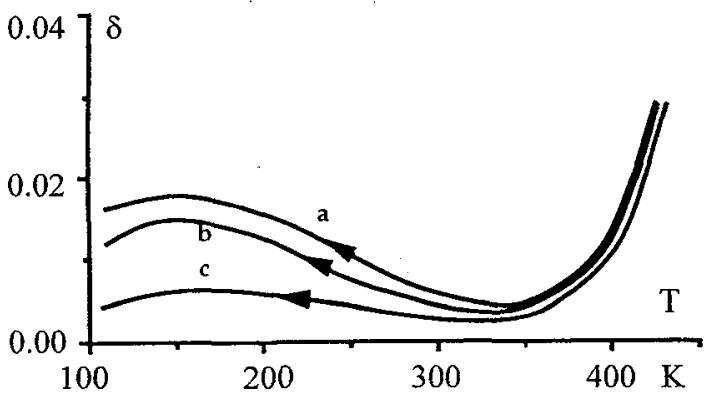

Figure 3 : Logarithmic decrement $\delta$ vs $T$ for various cooling rates $|\dot{T}|=200,100,50,0 \mathrm{~K} / \mathrm{h}$ for curves $\mathrm{a}, \mathrm{b}, \mathrm{c}$, $\mathrm{d}$, respectively. for Al-7075/15 vol. $\% \mathrm{SiC}$; shear strain amplitude $510^{-6}$; frequency $f=0.3 \mathrm{~Hz}$.

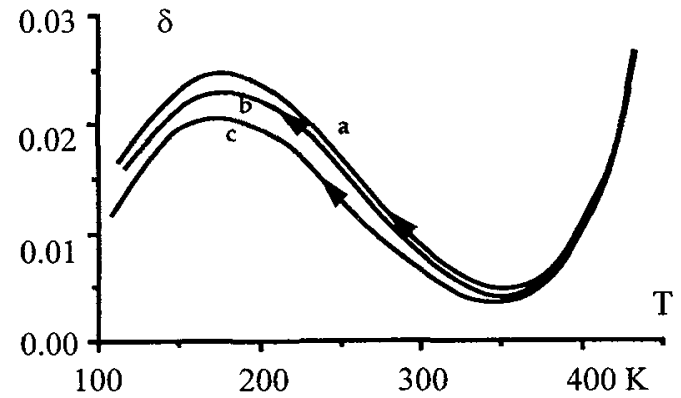

Figure 4 : Logarithmic decrement $\delta$ vs $T$ for various shear strain amplitudes $\gamma_{0}=110^{-6}, 510^{-6}, 110^{-5}$ for curves a, b, c, respectively. for Al-7075/15 vol.\% SiC ; cooling rate $|\mathrm{T}|=200 \mathrm{~K} / \mathrm{h}$; frequency $f=0.3 \mathrm{~Hz}$. 
Furthermore, in order to compare the above results obtained during cooling, with those corresponding with quasi-isothermal conditions, the temperature was decreased by steps of $20 \mathrm{~K}$ and then the temperature was kept constant during 30 min before each measurement. The discontinuous spectrum thus obtained, labelled $\mathbf{d}$ in Fig. 3, shows that the high level of I.D. measured previously on continuously cooling the material has completely disappeared. This means that the observed phenomenon has a transient character. This conclusion has been confirmed by the fact that the I.D. intensity decreases rapidly from the level given by curve a to the level indicated by curve $\mathbf{d}$ when cooling is stopped and $\mathrm{T}$ is maintained constant. Besides, note that, on the one hand, the L.T.M. was perfectly reproducible when the specimen was reheated up to $430 \mathrm{~K}$ and tested again and, on the other hand, it did not appear for the unreinforced matrix, whatever the cooling rate.

The influence of the oscillation period of the pendulum (i. e. inverse of frequency) on the L.T.M. is similar to that of the cooling rate, namely the intensity of the L.T.M. is strongly decreased as the period is decreased [24]. In contrast, a weaker influence of the oscillation amplitude is observed. Thus, it can be seen in Fig. 4 that the I.D. at the maximum is only slightly decreased as the surface shear strain amplitude is increased from $10^{-6}$ to $10^{-5}$. Moreover, the intensity of the L.T.M. is strongly dependent on the microyield stress of the matrix, i.e. the L.T.M. is greater the lower the microyield stress of the matrix [25].

\section{3 - I.D. during a thermal cycle}

Various complementary experiments were also performed in order to characterize completely the L.T.M.. The details of these experiments are presented in reference [24]. Among these, it is worth mentioning the behaviour during complete and incomplete thermal cycles. First, these experiments show that the I.D. in the low temperature domain is much smaller on heating than on cooling the specimen. Second, when an incomplete reheating is carried out, for instance the heating stage is interrupted at $300 \mathrm{~K}$, the subsequent I.D. spectrum on cooling is markedly different from the initial one.

Moreover, it should be noted that most of the above listed basic properties of the phenomenon have also been observed in other microheterogeneous materials such as $\mathrm{Al}-12 \% \mathrm{Si}$ alloys [17], $\mathrm{Al} / \mathrm{SiC}$ particulate composites [18], and $\mathrm{Al}-4 \% \mathrm{Cu} / \mathrm{Al}_{2} \mathrm{O}_{3}$ short fibre composites [19].

\subsection{Influence of volumetric fraction of particles}

The influence of the volumetric fraction of particles on the I.D. spectrum measured on cooling is shown in Fig. 5 in the case of the $\mathrm{Al}$ based composite. The intensity of the above L.T.M. is increased as the volumetric fraction up to $12 \%$. In contrast, the phenomenon seems to be saturated for a concentration of 20 vol.\% SiC particles.
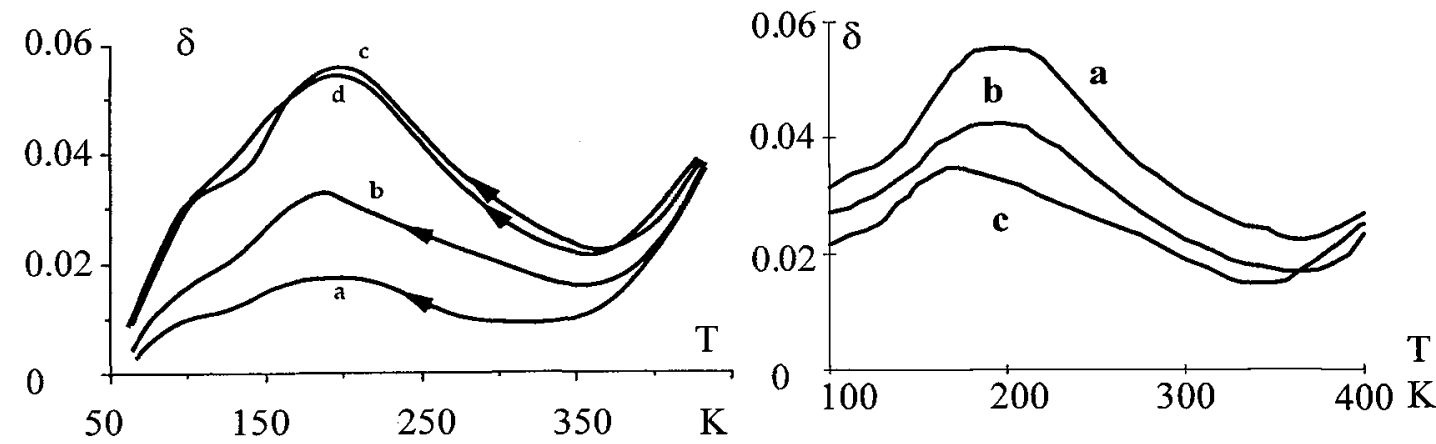

Figure 5 : Logarithmic decrement $\delta$ vs T for various Figure $6:$ Logarithmic decrement $\delta$ vs $\mathrm{T}$ for various vol. $\%$ of $\mathrm{SiC}$ particles in a pure $\mathrm{Al}$ matrix : $4,8,12,20$ interface preparations of $\mathrm{Al} / \mathrm{SiC}$ composites : a) nonvol. \% for curves $\mathrm{a}, \mathrm{b}, \mathrm{c}, \mathrm{d}$, respectively; cooling rate $|\mathrm{T}|$ oxidized $\mathrm{SiC}$ particles, b) oxidized $\mathrm{OX} 1$, c) oxidized $=200 \mathrm{~K} / \mathrm{h} ; \gamma_{0}=510^{-6} ; \mathrm{f}=0.6 \mathrm{~Hz}$. $\mathrm{OX} 2 ; \gamma_{0}=510^{-6} ;|\hat{\mathrm{T}}|=200 \mathrm{~K} / \mathrm{h} ; \mathrm{f}=0.35 \mathrm{~Hz}$.

\subsection{Influence of interfacial region}

The ID spectra of the three M.M.C.s prepared with SiC particles previously oxidized under various conditions have been obtained on cooling each of them from $430 \mathrm{~K}$. The low temperature part of these spectra is shown in Fig. 6. It appears clearly that the intensity of the low temperature peak depends on these oxidization conditions. In order to understand the differences which appear in the ID spectra, 
Transmission Electron Microscopy (T.E.M.) observations of the particle-matrix interfacial region have been carried out. Two examples of such observations are given in Fig. 7. An interfacial layer between particle and matrix is clearly seen in the materials elaborated with oxidized SiC particles (Fig. 7-b), but it does not in the standard material (non-oxidized particles, Fig. 7-a). From these bright field T.E.M. observations, the overall thickness of the interfacial layers have been evaluated to be of order of $10 \mathrm{~nm}$ and $20 \mathrm{~nm}$ for OX1 and OX2 composites, respectively. Furthermore, in the interfacial layer (case of the oxidized particles), the existence of at least a third phase of amorphous nature has been proved from diffraction and dark field observations. More details about these observations can be found in [26].

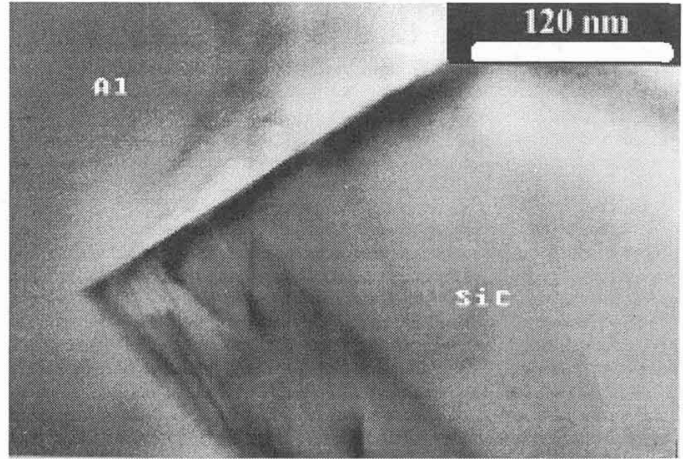

a)

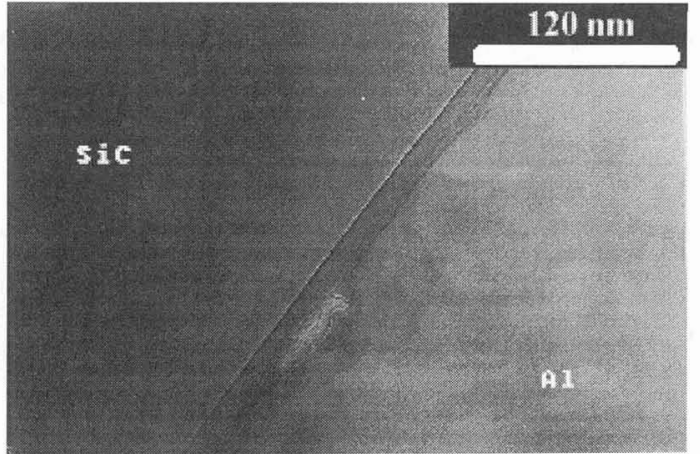

b)

Figure 7 : T.E.M. micrographs of the Al-SiC interface with a) non-oxidized particles and b) OX2 particles.

\section{COMPUTATIONAL MODELLING OF THE TRANSIENT INTERNAL DAMPING}

\subsection{Basis of the I.D. model}

In microheterogeneous materials, I.D. may result from contributions due to the intrinsic properties of each phase and from contributions linked with the interaction between particles and matrix. The proposed model aims at accounting for I.D. responsible for the $\dot{T}$ effect. This effect has been shown to result from the presence of $\mathrm{SiC}$ particles. Furthermore, since $\mathrm{SiC}$ particles are expected to remain perfectly elastic the $\dot{T}$ effect can only result from the particle-matrix interaction, i.e. dislocation movernents induced around $\mathrm{SiC}$ particles to relieve the stress concentration of thermal origin. More definitely, the $\dot{\mathrm{T}}$ effect is ascribed to the coupling between the local microplastic strain of thermal origin and the oscillating shear stress produced by the pendulum oscillations. The calculation of the resulting I.D. can been carried out in three steps. First, a detailed study of movements of the dislocation segments subjected to both the thermal stress and the alternating shear stress is carried out. Second the shear strain rate of an appropriate volume associated to a particle in the composite is deduced from the above movements. Third, the dissipated energy and the logarithmic decrement are calculated. These steps are described briefly in what follows.

\subsection{Dislocation movements}

The model for dislocation punching out under pure thermal loading, presented in section 2 , has to be modified to account for the applied shear stress, $\tau$, in a torsion pendulum. The situation is schematized in Fig. 8. Some dislocation segments such as $\mathrm{AA}^{\prime}$ are not sensitive to the pendulum shear stress. On the contrary, segments such as $\mathrm{BB}^{\prime}$ are distorted. Indeed, during a half-period, the pendulum shear stress acts in the same sense as the thermal stress on side B of the loop and consequently its movement is accelerated, while these stresses are opposing each other for side B', such that B' movement is slowed down or stopped. Since the shear stress induced by the pendulum oscillation is alternate, during the next half-period, roles of B and B' are inverted. As a result of this unsymmetrical displacement of dislocation loops of B and B' types, an inelastic shear strain $\gamma$ is induced by the applied shear stress. It is the source of I.D. in the M.M.C. specimen.

For the sake of clarity the approach we use to determine the dislocation motion is described only for one group of dislocation. The details of the calculation, including its extension to several groups of dislocation can be found in the work of Girard [24]. In the following treatment the basic assumptions presented in section 2 for the punching out model are again used. In addition, it should be noted that the line tension 
which could act as a restoring force when the loops are distorted is neglected. That is the movements of the various parts of a dislocation loop are treated as if they were independent from each other.This approximation is quite sound for the $7075 \mathrm{Al}$ matrix, which exhibits a high level of friction stress, but it is less justified in the case of the pure Al matrix.

First, consider the specimen without any applied shear stress. On cooling, the thermal driving shear stress (see section 2), which acts on a dislocation that is in equilibrium at a distance $x^{0}$ (defined by eq. 2 ) from the particle-matrix interface, is hereafter noted $\tau_{\mathrm{d}}\left(\mathrm{x}^{0}, T\right)$. Then, when the pendulum is operating, the new equation for the dislocation position has to be written :

$$
\tau_{d}(x, t)+\tau_{0} \sin \omega t=\tau_{f}(x)
$$

where $\tau_{0}$ is the amplitude of the shear stress induced by the pendulum oscillations, $\omega$ is the angular frequency of the oscillations and $\mathrm{x}$ is the resulting position of the dislocation loop.

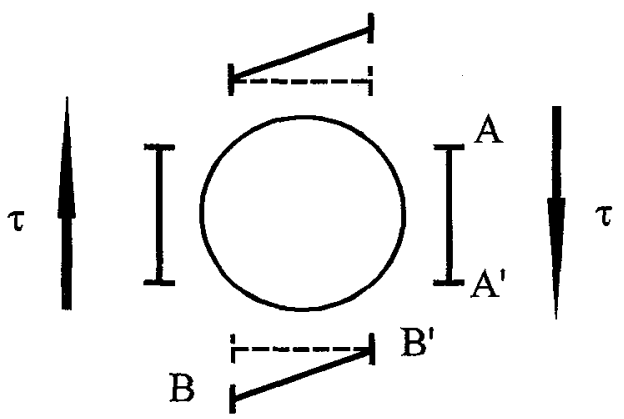

Figure 8 : Schematic of the shear stress effect on the dislocations loops punched out under the action of the thermal driving stress

Provided that $\tau_{0}<<\tau_{\mathrm{d}}$, the actual position $\mathrm{x}$ reached by the dislocation when the pendulum is operating remains close from that induced by the thermal stress field alone, namely $x^{0}$. Then, $x$ can be derived from $\mathrm{x}^{0}$ by expanding $\tau_{\mathrm{d}}(\mathrm{x}, \mathrm{t})$ at the first order vs $\mathrm{x}$, that is :

$$
\tau_{\mathrm{d}}(\mathrm{x}, \mathrm{t})=\tau_{\mathrm{d}}\left(\mathrm{x}^{0}, \mathrm{t}\right)+\left(\mathrm{x}-\mathrm{x}^{0}\right) \frac{\partial \tau_{\mathrm{d}}}{\partial \mathrm{x}}
$$

Dropping $\tau_{\mathrm{d}}$ into eq.(3) and using eq.(2) lead to the following expression for the perturbation of position, $\mathrm{x}-\mathrm{x}_{0}$, induced by the pendulum stress :

$$
x-x^{0} \cong-\tau_{0} \sin \omega t /\left(\frac{\partial \tau_{d}}{\partial x}\right)
$$

Note this expression is only valid when the resulting dislocation velocity is increased by the pendulum stress. Indeed, a velocity decrease corresponds to a situation for which the thermal and pendulum stresses are in opposition to each other. In that case the corresponding dislocation remains immobile because the driving stress becomes lower than the friction stress. Note that in this work, since $\tau_{0}<\tau_{\mathrm{d}}$, the situation for which the total driving stress would fall below $-\tau_{\mathrm{f}}$ has not been considered.

\subsection{Shear strain rate}

A local shear strain rate can be easily deduced from the above calculated dislocation movements. For that purpose, the composite material is idealized in terms of an array of identical cells containing each a single spherical particle. Hence, the volume $V_{r}$ associated to each cell is given by $V_{r}=4 \pi a^{3} /(3 \mathrm{f})$, where $a$ is the radius of particles and $f$ their volumetric fraction. Then, the Orowan's law can be applied to this volume $V_{r}$. The cell plastic shear strain, $\gamma_{\mathrm{P}}$, is deduced from the differential movement of the dislocation segments deduced from eq. (5), that is :

$$
\gamma_{\mathrm{P}}=\lambda \mathrm{Lb}\left(\mathrm{x}-\mathrm{x}^{0}\right) / \mathrm{V}_{\mathrm{r}}
$$

where $\mathrm{L}$ is the length of dislocation line in the considered dislocation group, $\mathrm{b}$ is the burgers vector and $\lambda$ is an orientation factor of order of $1 / 3$, accounting for the fact that only a part of the punched out dislocations are well oriented to feel the applied shear stress.

\subsection{I.D. computation}

In the final step of the calculation, the energy loss during one oscillation cycle of the specimen, $\Delta \mathrm{W}$, is deduced from the preceding steps by integrating the work done by the local applied stress over the whole volume of the specimen, during a period of oscillation. For a parallelepiped specimen, with a thickness 
much smaller than its width, and for a torque applied along its longitudinal axis, the volume integral comes to a one-dimension integral over the shear stress, which varies vs the distance between the considered point and the symmetry plane parallel to the largest faces of the specimen :

$$
\Delta \mathrm{W}=\frac{2 \mathrm{~V}}{\tau_{\max }} \tau_{0}^{\tau_{\max }} \int_{\dot{\gamma}_{\mathrm{P}} \mathrm{dtd} \tau}
$$

Then, $\delta_{\dot{T}}$ is deduced from the well-known expression $\delta=\Delta \mathrm{W} / 2 \mathrm{~W}$, where $\mathrm{W}$ is the maximal elastic energy stored during 1 cycle of oscillation.

Finally, in order to simulate a complete I.D. spectrum $\delta_{\dot{T}}$ vs $T$, the above described calculation has to be repeated after each temperature decrement. It should be recalled that several dislocation groups are punched out throughout the cooling process, such that the interaction between the groups must be taken into account and their respective contributions must be summed.

\section{RESULTS OF COMPUTATION AND DISCUSSION}

\subsection{I.D. in M.M.C.s with a perfect interface}

The above model is expected to be well suitable for describing the properties of M.M.C.s prepared with non-oxidized particles for which the T.E.M. examinations of the interfaces have not revealed any defect. An example of spectrum $\delta_{\dot{T}}$ vs T computed under such conditions is shown in Fig. 9. Furthermore, in order to make a sound comparison with experimental data, it is necessary to extract $\delta_{\dot{T}}$ from the measured $\delta$ vs T spectrum. For that purpose, following Girard et al [18], the experimental I.D. can be shared in two parts : one dependent on $\dot{\mathrm{T}}$ and another one independent of $\dot{\mathrm{T}}$, hereafter noted $\delta_{\mathrm{B}}$. Hence, $\delta_{\mathrm{B}}$ is readily obtained from the measurements performed under isothermal conditions. In turn, under the assumption that $\delta_{\mathrm{B}}$ is not affected by the $\dot{\mathrm{T}}$ effect, the contribution $\delta_{\dot{\mathrm{T}}}$ can be determined by subtracting $\delta_{\mathrm{B}}$ from the measured coefficient $\delta$. A series of experimental values of $\delta_{\dot{T}}$ thus obtained with the same experimental parameters as for computation (corresponding to spectrum a of Fig. 2), has been also plotted in this Fig. 9. It appears that the agreement between experimental and computed values is fairly good. It should be noted that such an agreement between computed and measured I.D. is not so good for the $\mathrm{Al} / \mathrm{SiC}$ composites as for the $7075 \mathrm{Al} /$ composite. This can be explained by the fact that the conditions of validity of the model, especially non overlapping plastic zones between neighbouring particles and negligible effect of line tension, is not so well satisfied for the former composite. This explanation is well supported by the saturation of I.D. that has been observed for the $\mathrm{Al} / \mathrm{SiC}$ composite with increasing the volume fraction of reinforcing particles (see Fig.5).
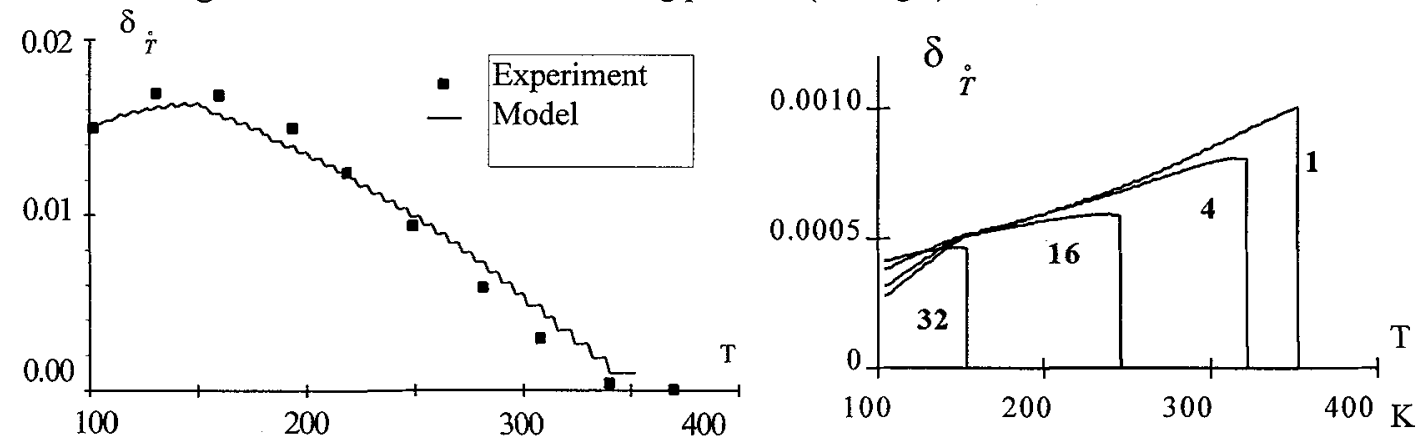

Figure 9 : Logarithmic decrement $\delta_{\Upsilon}$ vs $\mathrm{T}$ : computed Figure 10: Individual contribution of dislocation spectrum (solid line) and experimental points derived from groups $\mathrm{N}^{\circ} 1,4,16,32$ to I.D. vs temperature on spectrum a of Fig. 2 (a). cooling a Al-7075/SiC composite.

Furthermore, in order to understand the physical origin of the observed L.T.M., the contribution of each group of emitted dislocations has been computed separately vs T. Some typical evolutions are shown in Fig. 10. It appears clearly that the emission of one group contributes to a step like increase of the I.D., but this contribution is gradually decreased with further decreasing the temperature. Moreover, the magnitude of the step like increase is also decreased with decreasing $\mathrm{T}$. These evolutions are mainly due 
to the reduction of the dislocation mobility at low temperature, that is the increase in $\tau_{\mathrm{f}}$, at low temperature. Hence, the presence of the L.T.M. on cooling the system can be understood as resulting from the competition of two processes : an increasing number of dislocation groups and a decreasing contribution of previously emitted groups. In other words, the former process prevails above the temperature of the maximum of $\delta_{\mathrm{T}}$, whereas the latter prevails below this temperature.

Besides, in order to completely investigate the influence of the various experimental parameters, ID spectra can be computed under various conditions, over ranges more extended than those possible experimentally. The results of such an investigation is illustrated in what follows for the influence of $\dot{T}$, in the case of the Al-7075/15 vol\% SiC. Thus, the intensity of the maximum of $\delta_{\dot{T}}$ has been plotted vs $\dot{T}$ in Fig. 11 for cooling rates ranging from $10 \mathrm{~K} / \mathrm{h}$ to $2000 \mathrm{~K} / \mathrm{h}$. This figure shows that the intensity of the L.T.M. should go through a maximum vs temperature. The corresponding experimental values have been also reported in this plot, thus confirming the very good agreement between computed and experimental values. Unfortunately, the presence of the maximum cannot be reproduced experimentally because this would require cooling rates faster than $500 \mathrm{~K} / \mathrm{h}$. Furthermore, it is worth mentioning the computed predictions fit also very well the frequency and the strain amplitude dependences of the L.T.M. intensity. Finally, in order to understand the origin of such maxima, a detailed study of the dislocation motions throughout an oscillation has been carried out. The details of the study can be found in reference [24]. This study shows that at very high cooling rates dislocation motions are continuous because they are mainly controlled by the thermal driving stress. As a consequence, the work done by the applied stress during the half-cycle for which the applied and thermal stresses act in the same sense is almost completely recovered during the next half-cycle for which the applied and thermal stresses oppose each other. Hence, the I.D. is very low. In contrast, at very low cooling rates, dislocation motions are controlled by both the thermal and applied stresses, thus leading to discontinuous dislocation motions, whose magnitude decreases with decreasing the cooling rate. Hence, again a low I.D. level is expected. Between- these limit situations, I.D. goes through a maximum as the amplitude of the dislocation motion is maximum during the half-cycle for which the applied and thermal stresses act in the same sense, and the dislocations are stopped during the next half-cycle when they oppose each other, so that the work done during the first half-cycle is not recovered, i. e. mechanical energy is dissipated.
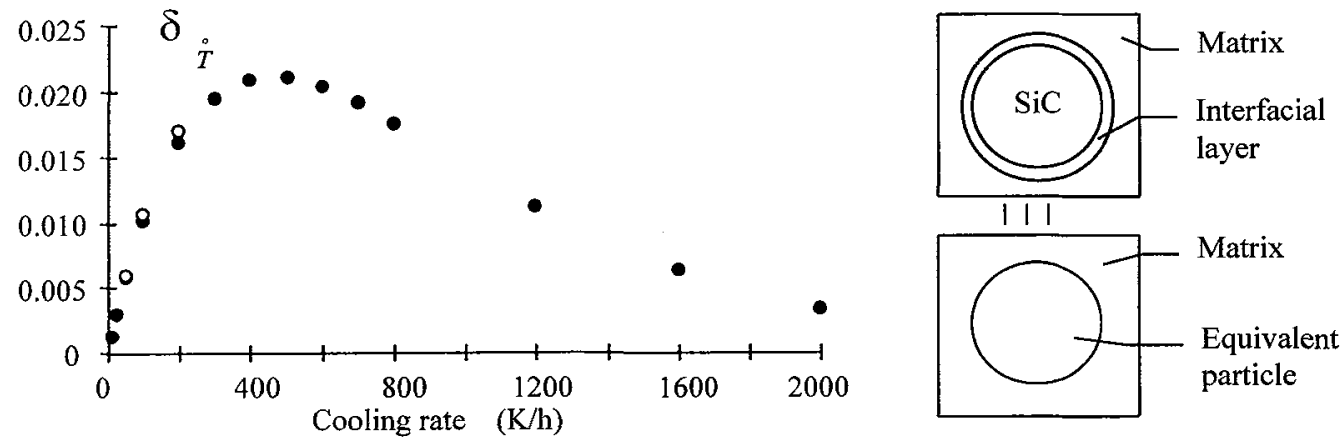

Figure 11 : Computed $(\bullet)$ and experimental $(0)$ values of the Figure 12 : Schematic of the equivalent L.T.M. intensity vs cooling rate $|\dot{T}|$ for the Al-7075/15 vol.\% particle used to simulate an elastic interfacial composite with experimental conditions of Fig. 2.

layer in the computational modelling of I.D.

\subsection{I.D. in M.M.C.s with oxidized particles}

Our experimental results indicate the ID peak level is decreased when an interfacial layer is produced between the $\mathrm{SiC}$ reinforcement and the aluminium matrix and, also, the thicker this layer is, the more the ID is decreased. This suggests that this layer would act upon the mechanisms liable for ID, i.e. the emission and the movements of the dislocations punched out from the particle-matrix interface for accommodating the strain mismatch of thermal origin between particle and matrix. In addition, from the above numerical computation, it should be kept in mind that ID is increased approximately as the number of emitted dislocations and it is decreased when the friction stress of the matrix, $\tau_{f}$, is increased. Then, considering the experimental ID levels (see Fig. 6) at the peak temperature (200 K) shows that ID 
is decreased of about $25 \%$ for the OX1 composite in comparison with the material reinforced by nonoxidized particles. This decrease amounts to about $30 \%$ for the OX2 composite. Then, two mechanisms could explain this phenomenon : i) the number of emitted dislocations is decreased because a part of the thermal expansion mismatch is accommodated by an elastic (or elasto-plastic) strain of the interfacial layer ; ii) $\tau_{\mathrm{f}}$ is increased in the adjacent Al matrix by some physico-chemical reaction between the interfacial phase and aluminium. Considering the former hypothesis indicates that the number of emitted dislocations would be decreased by about $25 \%$ for OX1 and $30 \%$ for OX2 (each dislocation has almost the same contribution to ID). So, to evaluate the soundness of such a mechanism, the above model cannot be used just as it is, i.e. the presence of an interfacial layer must be accounted for. For that purpose, assuming the interfacial layer is elastic and perfectly bounded to both the matrix and the particle, the overall elastic layer/particle system has been replaced by an equivalent single particle having the same elastic behaviour as the overall system. Then, all the parameters involved in the model are known - i. e. mechanical properties of the aluminium matrix and $\mathrm{SiC}$ particles as well as thermal ones, thickness of the interfacial layer - except the Young's modulus of the interfacial layer. Hence, in the numerical computation, this latter parameter can be used as an adjustable parameter in order to reach a number of emitted dislocations about $25 \%$ and $30 \%$ lower, for OX1 and OX2, respectively, than the number computed under the assumption of a perfect interface. This occurs for a Young's modulus of about $110 \mathrm{MPa}$ and $150 \mathrm{MPa}$, respectively. But, these values seem to be too low to be realistic. Therefore, the assumption of a significant contribution of the layer to an elastic accommodation of the strain incompatibilities between particle and matrix has to be ruled out. Note the method we have used is strictly valid only for studying the case of an elastic layer, but the case of an elasto-plastic layer can be approached as well by considering the concept of secant modulus. However, even in the case of secant moduli, the above numerical values seem unrealistic since they would mean a very low yield stress and a very small strain hardening in the interfacial layer.

So, these arguments lead us to the conclusion that only a decrease in the mobility of the dislocations in the vicinity of the reinforcing particles could explain the differences in $\mathrm{DD}$ between the standard composite and those elaborated with oxidized particles. As above mentioned such a decrease in the dislocation mobility could be induced by some physico-chemical reaction between the oxidized layer and the aluminium matrix during the elaboration process. This mechanism is not unlikely since, according to the Hamman's model, ID is much sensitive to dislocation mobility in the interfacial region. Indeed, decreasing the mobility of dislocation also induces a reduction in the number of the punched out dislocations, which is expected to enhance the phenomenon.

\section{CONCLUSION}

It has been shown that, among the various specific I.D. properties exhibited by M.M.C.s, the transient I.D. phenomenon observed on cooling (or heating) provides fruitful information dealing with the thermal internal stress state linked with the SiC-matrix C.T.E. mismatch and the interfacial region. In order to understand completely the phenomenon and to gain more benefit from the experimental data, a computational modelling of the phenomenon has been carried out on the basis of dislocation movements induced by the thermal stress field due to the C.T.E. mismatch. This approach has enabled us to describe and understand the properties of the transient I.D. : existence of a broad maximum on cooling, influence of cooling rate, frequency and strain amplitude. Furthermore, the transient I.D. has been shown to be quite sensitive to changes in the properties of the interfacial region caused by the oxidization of SiC particles prior to the elaboration of the composite. In brief, the I.D. measurements and the proposed analysis show that this method is quite useful for characterising thermal stresses and properties of the interfacial region in M.M.C.s, but further modelling works remain to be done in order to account for the non perfect interfaces.

\section{ACKNOWLEDGEMENTS}

This work was supported by the Aérospatiale Society (Suresnes laboratory), which is gratefully acknowledged. We are also very grateful to $\mathrm{P}$. Fondères (Villetaneuse University) for elaborating the $\mathrm{Al} / \mathrm{SiC}$ composite. 


\section{REFERENCES}

[1] Withers P.J., Stoobs W. M. and Pedersen O.B., Acta Metallurgica et Materialia, vol. 37, 11, (1989), p. 3061-3084.

[2] Mura T., " Micromechanics of Defects in Solids » Second Edition, Dordrecht (NL), Martinus nijhoff Publishers, 1987, 587p.

[3] Volgelsang M., Arsenauld R.J., and Fisher R. M., Metallurgical Transactions A, 17 A, march (1986), p. 379-389.

[4] Prangnell and Stobbs «The Effect of Internal Stresses on Precipitation Behaviour in Particulate Reinforced Al matrix MMCs » in Proc. of the $12^{\text {th }}$ RISO International Symposium on Materials Science, Roskilde Denmark 1991, N. Hansen, D. Jensen, T. Leffers, H. Lilholt T. Lorentzen, A.S. Pedersen Eds, (1991), p. 603.

[5] Strangwood M., Hippsley C.A., and Lewandowski J.J., Scripta Metallurgica et Materialia, vol. 24, (1990), p. 1483-1487.

[6] Eshelby, J. D., Prog. Solid Mech., 2, 89, 1961, p. 87-140.

[7] Dunan D.C. and Mortensen, Acta Metallurgica et Materialia, vol. 39, 2, (1991), p. 127-139.

[8] Shibata S., Taya M., Mori M. and Mura T., Acta Metallurgica et Materialia, vol. 40, 11, (1992), p. 3141-3148.

[9] Hamann R. and Fougères R., «A model for the microplastic strains of particulate reinforced M.M.C.s " in Proc. of the $12^{\text {th }}$ RISO International Symposium on Materials Science, Roskilde Denmark 1991, N. Hansen, D. Jensen, T. Leffers, H. Lilholt T. Lorentzen, A.S. Pedersen Eds, (1991), p. 373.

[10] Hull and Bacon

[11] Perez R. J., Zhang J., Gungor M. N., and Lavernia E.J., Metallurgical Transactions A, 24 A, march (1993), p. 701-712.

[12] Hartman J. T., Keene K. H., Armstrong R. J. and Wolfenden A., Journal of Metals, april (1986), p. 33-35.

[13] Rivière A., Fadel L., and Woirgard J. , "High Temperature Damping in Metal Matrix Composites », Intergranular and Interphase Boundaries in Materials, Thessaloniki, june 21-26, Ph. Komninou and A. Rocher Eds, Trans. Tech. Publications (1993), p. 671-674

[14] Girard C., Fougères R., Vincent A., Journal of Alloys and Compouds, 211/212, (1994), p. p. 169172.

[15] Delorme J. F., Schmid R., Robin M., and Gobin P. F., J. de Physique, 32 C2, (1971), p.101-105.

[16] Postnikov V.S., Gridnev S.A., Darinskii B. M., and Sharshakov I.M., Nuevo Cim., 33B, 1 (1976), p. 324-337.

[17] Zhou X., Fougères R., and Vincent A., J. Phys. III France 2, Novembre (1992), p. 2185-2201.

[18] Girard C., Lormand G., Fougères R. and Vincent A., Scripta Metallurgica et Materialia, vol. 28, (1993), p. 1047-1052.

[19] Parrini L. and Schaller R., Acta Metallurgica et Materialia, to be published (1996).

[20] Okabe M., Mori T. and Mura T., Philosophical Magazine A, 1981, vol. 44, No , (1981), p. 1-12.

[21] Parrini L. and Schaller R., Metallurgical and Materials Transactions A, vol. 26A, june (1995), p. $1457-1460$.

[22] Milligan K. B. and Kinra V. K., Mechanics Research Communications, vol. 20 (2), (1993), p. 137142.

[23] Milligan K. B. and Kinra V. K., Journal of Applied Mechanics, vol. 62, june (1995), p. 441- 449.

[24] Girard C., Ph. D. Thesis, Lyon, (1994), 276 p.

[25] Vincent A., Girard C., Lormand G., Zhou X., and Fougères R., Materials Science and Engineering A, vol. 164, 1-2, (1993), p.327-331.

[26] Durieux S., Esnouf C., Maire E., Fondère J.P., Fougères R., Lormand G., and Vincent A., Scripta Metallurgica et Materialia, to be published (1996). 